\title{
Controlled intervention to compare the efficacies of manual pressure release and the muscle energy technique for treating mechanical neck pain due to upper trapezius trigger points
}

This article was published in the following Dove Press journal: Journal of Pain Research

\author{
Richa Kashyap' \\ Amir lqbal ${ }^{2}$ \\ Ahmad H Alghadir ${ }^{2}$ \\ 'Department of Physiotherapy, \\ Prakash Institute of Physiotherapy \\ Rehabilitation and Allied Medical \\ Sciences, Chaudhary Charan Singh \\ University (Meerut), Uttar Pradesh, \\ India; ${ }^{2}$ Rehabilitation Research Chair \\ College of Applied Medical Sciences, \\ King Saud University, Riyadh, Saudi \\ Arabia
}

Purpose: This study aimed at comparing the clinical efficacies of two manual therapies to determine the most beneficial result-oriented physiotherapeutic approach for treating nonspecific neck pain due to myofascial trigger points (MTrPs).

Methods: This was a randomized, controlled pretest-posttest experimental study that compared manual pressure release (MPR), the muscle energy technique (MET), and a control condition. These techniques were compared using a convenience sample of 45 female participants with neck pain due to MTrPs (mean age $\pm \mathrm{SD}=21.49 \pm 3.66$; age range $=18-30$ years). The visual analog scale, pressure pain threshold, Neck Disability Index Questionnaire, and a standardized measuring tape were used to assess the participants' neck pain, muscle tenderness, functional disability due to neck pain, and range of neck rotation, respectively, at baseline (day 0), day 1, and day 5 postintervention and at days 10 and 15 during follow-up. All groups were given postural advice and at-home neck exercises. Repeated-measures ANOVA and one-way ANOVA were used to analyze the data.

Results: The within-group analyses showed significant improvement $(P<0.05)$ in all outcome measures at days 1 and 5 postintervention and at days 10 and 15 during the follow-up for all groups. The between-group analyses confirmed nonsignificant differences $(P>0.05)$ between all groups for all variables.

Conclusion: MPR and the MET are equally effective for reducing pain and muscle tenderness and for improving neck disability and range of rotation in patients with nonspecific neck pain. Furthermore, advice promoting postural correction can be an adjunct to physiotherapeutic interventions for reducing neck pain and its symptoms. A combination of these manual therapies with postural advice might be a good treatment option for nonspecific pain in physiotherapy clinics Keywords: neck pain, manual pressure release, muscle energy technique

\section{Introduction}

According to epidemiological surveys, $\sim 45 \%-54 \%$ of the general population worldwide is affected by mechanical pain at some time during their lives, and it often progresses to medical treatment, absenteeism from work, and even severe disability. ${ }^{1,2}$ Among workers in the information technology industries, 23\%-33\% report neck pain and $7 \%-17 \%$ have reduced neck movement. ${ }^{3}$ Chronic neck pain is further associated with a greater activation of accessory neck muscles during repetitive upper limb tasks due to decreased neck muscle strength and restricted movements. ${ }^{4,5}$ Major sources of neck pain are direct trauma, impairment, degeneration, and derangement of intervertebral
Correspondence: Amir lqbal G95-I, Rehabilitation Research Chair, College of Applied Medical Sciences, King Saud University, PO Box 10219, Riyadh

I I433, Saudi Arabia

$\mathrm{Tel}+96614696010$

Fax+966I 4693589

Email ajamaluddin@ksu.edu.sa 
discs, ligaments, muscles, facet joints, dura, and nerve roots. ${ }^{6}$ Nonspecific pain in the neck area, with or without radiation to the extremities, is generally a by-product of poor posture or long-term abnormal physiological loads. Physical deconditioning, lack of exercise, and frequent abnormal loading result in the formation of small nodular taut bands, termed "trigger points," in the musculature around the neck, causing a musculoskeletal imbalance in the upper quarter of the body. $^{7,8}$

Myofascial trigger points (MTrPs) are thought to be a common source of musculoskeletal pain and impairment, both local and referred. MTrPs are characterized as discrete foci, often palpable as a nodule, within taut bands of skeletal muscle that are tender upon palpation and produce characteristic referred pain and autonomic phenomena. Because practitioners often receive insufficient training and knowledge in this area, MTrPs are inadequately diagnosed and treated. Patients with both active and latent trigger points can present with complex clinical findings. The suboccipitalis, sternocleidomastoid, splenius capitis, and upper trapezius muscles most commonly harbor the trigger points primarily responsible for neck and head pain. ${ }^{9}$

Most current treatments for neck pain are based on anecdotal observation and historical management methods. ${ }^{10}$ Due to the elusive and multifaceted pathophysiology of neck pain, approaches to its treatment and the validation of such treatments are currently mostly empirical. ${ }^{11}$ Thus, biopsychosocial models and combined approaches are gaining popularity. ${ }^{12}$ The Philadelphia panel evidence-based practice guidelines for neck pain stated that therapeutic exercises are the only interventions that provide clinically meaningful benefits relative to control treatments. Traditionally, interventions implemented to manage neck pain due to MTrPs pain include cryotherapy, electrotherapy, thermotherapy, local anesthetics, dry needling, steroid injections, therapeutic ultrasounds, massage, and galvanic stimulation. ${ }^{8}$ However, most of these treatment methods lack evidence regarding their efficacies for deactivating trigger points and reducing neck pain. Manual therapy is more cost-effective for treating nonspecific pain than physiotherapy or care by a general practitioner. ${ }^{13}$

Manual pressure release (MPR), previously known as "sustained manual pressure," "ischemic compression," "inhibition," and "trigger point release," is one of the manual therapy techniques utilized for the treatment of trigger points. It is practiced by delivering bearably painful, persistent manual pressure, generally with the thumb or fingertip, against the tissue barrier of an MTrP. It is used to lengthen the sarcomeres of contraction knot present in the affected muscle fibers. It works through the principle of slow sustained stretch that produces lengthening in muscle fiber that results in ease of tightness and relief of pain in MTrP case. However, the muscle energy technique (MET), which is a direct, noninvasive manual therapy, is also used to normalize muscle length and increase range of motion, and its main objective includes relaxation of hypertonic musculature. When appropriate, subsequent stretching of the muscles can also be provided. It differs from MPR in using the principle of postisometric stretching for lengthening the sarcomeres of contraction knot present in the affected muscles, which reduces the tension in muscle fibers and thus provides relief of pain in patients with MTrPs.

Most research performed to date has supported the efficacy of MPR in treating trigger points. Very few studies have incorporated MET as an effective tool, and the long-term benefits of these techniques have yet to be determined. The objectives of this study were to assess and compare these two manual therapy techniques and to determine the efficacy of each therapy for treating trigger points. Clear connections have been established between MTrPs and a wide range of pain problems and sympathetic aberrations. ${ }^{14}$

Muscle tension results in muscle pain via prolonged contraction of intrafusal muscle fibers, which occurs through both the activation of gamma motor neurons and increased sympathetic nerve activity. ${ }^{14,15}$ Using both MPR and MET, we offer an opportunity for neurological and musculoskeletal resetting to occur. For this reason, this study aimed at comparing the clinical efficacies of these two manual therapies to determine the most beneficial result-oriented physiotherapeutic approach to resolve neck pain due to trigger points. It was hypothesized that either MPR or MET would be more effective than a control condition for treating neck pain.

\section{Materials and methods \\ Participants}

A total of 45 participants were assessed for the presence of trigger points and screened according to the inclusion and exclusion criteria of the study. The participants were recruited from the Physiotherapy Department of Kailash Hospital, Noida. Prior to the start of the study, the purpose and procedures of the study were explained clearly to the participants, and written informed consent was obtained from them. Overall, the 45 participants were allocated equally into experimental group A, experimental group B, and control group $\mathrm{C}$, using an online randomization tool, Randomization.com (http://www.randomization.com). The inclusion criteria were the following: female between 18 and 30 years 
of age, mechanical neck pain localized to the cervical or bilateral scapular regions, the presence of one or two trigger points, willingness to participate, and no clinical treatment for neck pain within the past month. The exclusion criteria were the following: a diagnosis of fibromyalgia syndrome according to the American College of Rheumatology; ${ }^{16}$ a history of neck and shoulder surgery in the past year; clinical evidence of myelopathy, radiculopathy, stenosis, thoracic outlet syndrome, vestibulobasilar artery syndrome, whiplash injury, infection, systemic disorder, migraine, or inflammatory arthritis in the cervical region; and poor cooperation.

Diagnostic criteria for $\mathrm{MTrPs}^{17}$ were the following:

1. Presence of a palpable taut band in the skeletal muscle;

2. Presence of a hypersensitive spot in the taut band;

3. Local twitch response provoked by snapping palpation;

4. Reproduction of the typical referred pain pattern in response to the compression of tender spots; and

5. Spontaneous presence of the typical referred pain pattern.

If only four criteria were satisfied, the trigger points were considered latent, and if all were satisfied, the trigger points were considered active.

\section{Study design}

This study was based on a pretest-posttest experimentalcontrol group design. Each group had 15 participants. MPR was performed for group A, whereas group B received the $\mathrm{MET}$, and group $\mathrm{C}$ served as the control group.

\section{Outcome measures}

The outcomes of the study were pain intensity, pressure pain threshold (PPT), cervical range of rotation (ROR), and functional status of the neck. Pain intensity was assessed using a visual analog scale (VAS), the PPT was obtained using a digital pressure algometer, cervical ROR was measured using a flexible measuring tape, and the functional status of the neck was evaluated using the Neck Disability Index (NDI) Questionnaire. The VAS is a subjective pain scale used to rate the level of pain intensity on a horizontal line of $10 \mathrm{~cm}$ that is marked with zero (0) at one end and ten (10) on the extreme of the other end. ${ }^{18}$ The test-retest reliability ( $r=0.71-0.94 ; P<0.001)$, construct validity, and feasibility of this scale show its suitability for measuring pain in musculoskeletal conditions. ${ }^{18,19} \mathrm{~A}$ pressure algometer is a cost-effective, clinically reliable, valid, and feasible tool that is used for the quantification of tenderness and treatment effects in myofascial and musculoskeletal pain. ${ }^{20,21}$ The intraclass correlation coefficient (ICC) is good (mean ICC
$=0.75$ ), and the test-retest reliability is excellent (mean ICC $=0.84) .{ }^{22}$ The NDI Questionnaire is a commonly used 10 -item self-report measure of disability resulting from neck pain. Each item describes a set of six statements related to pain or difficulties that affect the ability to manage the activities of daily living. ${ }^{23}$ The stable psychometric properties $(\alpha=0.92)$ of this questionnaire suggest that it offers an objective means for assessing the functional disability of patients suffering from neck pain. ${ }^{24}$

\section{Procedure}

After the initial screening and receiving informed consent, followed by allocation into groups, a baseline assessment of all variables was obtained from all the participants, and they received their group-specific intervention plan. Postintervention and follow-up data were collected on study days 1,5 , 10, and 15 as described in Figure 1.

\section{Measurements}

All the participants were given the NDI Questionnaire and instructed to mark the most suitable statement for each question related to their pain and difficulties that affect their ability to manage day-to-day life activities. The scores of each item were summed to find the final score for data analysis. The participants were instructed to be seated upright, facing forward on a chair with the back supported, and to keep their head in a neutral position to obtain the measurements for all physical variables. A flexible measuring tape was used to measure the cervical rotation in centimeters. The participants were asked to rotate their head actively to the extreme on the either side (left and right) while maintaining a consistent horizontal eye level. Then, the minimum distance between the tip of the acromion process and the midpoint of the chin was noted for both directions of neck rotation. The upper and middle trapezius muscle regions were exposed in a reasonable manner to mark the trigger points and measure the PPT level using the digital pressure algometer, as recommended by Fischer. ${ }^{21}$ The trigger point with the lowest PPT was chosen as the primary trigger point. The subjects were instructed to indicate when the sensation of pressure changed to a sensation of pain by saying "there" or "yes." Two repeated measurements were obtained by the same assistant, and the mean was used for the analysis. A gap of at least 1 minute was included between the two repeated measurements to reduce the possibility of sensitization. ${ }^{20,21}$ After obtaining preintervention data for the PPT, a second pressure of 2.5 $\mathrm{kg} / \mathrm{cm}^{2}$ was applied by a physiotherapist while the subjects were instructed to rate their pain on the VAS, to evaluate the 
Screening/evaluation

Recruited for the study (total of 45 patients)

Allocated into group A, group B, and group C (baseline data collected)

Group A received (manual pressure release+postural advice+active exercises)

Group B received (muscle energy technique+postural advice+active exercises)

Group $\mathrm{C}$ received (postural advice+active exercises)

Postintervention and follow-up data collected at their stipulated time

Days 1 and 5 postintervention and at days 10 and 15 during follow-up

Data analysis using SPSS Version 17.0

Figure I Flow diagram of the study protocol.

local pain evoked by the application of this amount of pressure. ${ }^{25}$ Preintervention (baseline) data were collected on day 1; postintervention data were collected on days 1 and 5; and follow-up data were collected on days 10 and 15 after the initiation of the study for all outcomes except the NDI. The NDI was documented preintervention on day 1 (baseline), day 5 (postintervention), and day 15 (during follow-up).

\section{Treatment interventions}

All the participants of experimental group A, experimental group $\mathrm{B}$, and control group $\mathrm{C}$ received the conventional interventions in the form of isometric neck exercises, postural advice, stretching, and gentle conditioning exercises for neck and shoulder girdle muscles. In addition, experimental group A received MPR technique, experimental group B received MET, whereas group $\mathrm{C}$ received conventional interventions only.

For MPR technique, the participants were instructed to be relaxed in a sitting position prior to the application of pressure. The examiner applied gradually increasing pressure to the MTrP until the participant reported moderate, but tolerable pain with a value of 7 out of 10 on the VAS (where $0=$ no pain, $10=$ maximum intolerable pain) and sustained that pressure until the participant reported that their pain decreased to a lesser value of 3 or 4 on the VAS. The examiner again slowly increased the pressure to restore the perceived pain level to the original value of 7 and maintained that pressure until a reduction of pain to a lesser value of 3 or 4 on VAS. Most often, the participants reported a reduction in tenderness (from 7 to 3 or 4 on VAS) after 20-30 seconds of the application of MPR.

For the MET, the participants were instructed to lie down in a supine position with the cervical spine in opposite lateral flexion to the area of the body being treated so that the upper trapezius muscle fibers were in a position that allowed them to be lengthened. ${ }^{19}$ Moderate isometric contraction ( $75 \%$ of maximal) of the upper trapezius muscles was elicited for a period of 5 seconds, followed by 3 seconds of relaxation while reaching to the new barrier. This maneuver was repeated four times in each session.

Isometric neck exercises taught to all the participants, and they were instructed to perform these exercises at home, in a neutral sitting position, thrice daily for 15 consecutive days. The sequence of exercises was as follows: shoulder circling in clockwise and counterclockwise directions; scapular retraction with cervical retraction; neck flexion, extension, and lateral bending/flexion on each side; and left and right rotation of the neck. Each exercise involved sets of 10 repetitions of maximum voluntary contraction with a progressive hold time of $3,5,7$, and 10 seconds, depending on the severity of pain and tolerance of the participants.

Postural advice was given to all the participants. They were advised that they should not sleep on a foam rubber pillow, as its springiness causes vibration that aggravates trigger point symptoms; should use a chair with proper back support and an arm rest of an appropriate height to provide elbow support while working at a keyboard and computer; should avoid leaning forward toward the screen of a computer or on a desk while 
writing for a prolonged period; and should avoid prolonged conversation on a telephone while holding the receiver by tilting the neck. Furthermore, the participants were advised to relieve muscle tension after every 20-30 minutes of work by getting up; stretching the arm, shoulder, neck, and back muscles; and walking to turn off the buzzer and reset the timer. They should avoid using a bra with tight and thin straps, as it produces an objectionable amount of pressure on the trapezius muscles. They should either avoid prolonged carrying of a bag on any one side of the shoulder or balance the hanging weight of the bag on both sides of the shoulder. Finally, they should perform gentle conditioning exercises for both the neck and shoulder girdle.

\section{Analysis}

SPSS Version 17 software was used to analyze the data. Repeated measures ANOVA and one-way ANOVA were used to analyze pairwise comparisons within and between groups, respectively, for all variables. Descriptive analysis was performed for the demographic variables. The level of significance $(\alpha)$ was set at 0.05 .

\section{Ethical considerations}

Ethical approval was granted for this study by the Institutional Review Board of Prakash Institute of Physiotherapy, Noida. This study maintained the participants' rights and was monitored to ensure appropriate research conduct. A written informed consent was obtained from all the participants prior to the inclusion in the study.

\section{Results}

Of the 51 participants, three dropped out with valid reasons, two failed to complete the intervention, and one complained of aggravated pain after the day 1 intervention and dropped out of the study. A total of 45 participants completed the study. The descriptive analysis of the baseline measurements of all variables revealed no significant differences $(P>0.05)$ between the three groups. Table 1 provides the means and SDs of all demographic characteristics in detail. The mean improvement was calculated as the difference between the means of the variables on different days for all groups. The results of the statistical analyses of the outcome measures (pain intensity [VAS], PPT, cervical ROR, and NDI) are explained in the following sections. Tables 2 and 3 present the within- and between-group comparisons, respectively.

\section{Pain intensity (VAS)}

The within-group analysis revealed a statistically significant improvement in pain intensity in group A (mean
Table I Demographic details of the groups

\begin{tabular}{|l|l|l|l|l|}
\hline Variables & Group A & Group B & Group C & P-values \\
\hline Numbers & 15 & 15 & 15 & 1.000 \\
\hline Age (years) & $21.27 \pm 3.86$ & $22.07 \pm 4.11$ & $21.13 \pm 3.00$ & 1.000 \\
\hline Weight (kg) & $51 \pm 8.72$ & $53.33 \pm 10.09$ & $48.2 \pm 7.76$ & 1.000 \\
\hline Height (cm) & $156.07 \pm 5.97$ & $157 \pm 4.05$ & $156 \pm 5.04$ & 1.000 \\
\hline Nature of works (for 7-8 hours) \\
\hline Mobile & 13 & $\mathrm{II}$ & 13 & 1.000 \\
\hline Sedentary & 2 & 4 & 2 & 1.000 \\
\hline
\end{tabular}

Note: The value is significant if $P<0.05$ and nonsignificant if $P>0.05$.

improvement $=-3.6 ; P<0.05)$, group $\mathrm{B}$ (mean improvement $=-3.87 ; P<0.05)$, and group $\mathrm{C}$ (mean improvement $=-3.4$; $P<0.05)$ when the follow-up scores at day 15 were compared with their respective baselines scores (VAS 15-VAS 0). In addition, all groups showed a significant improvement at follow-up day 10 (VAS 10-VAS 0) and even at day 5 postintervention (VAS 5-VAS 0) when compared with their respective baseline scores $(P<0.05$; Figure 2$)$. Interestingly, experimental group A had an insignificant improvement $(P>0.05)$, whereas experimental group B and even the control group $\mathrm{C}$ experienced significant improvements $(P<0.05)$ immediately postintervention on day 1 when compared with their baseline scores (VAS 1-VAS 0).

The between-group comparisons showed statistically insignificant differences in improvement $(P>0.05)$ for all time intervals except at follow-up day 10 , where group B was marginally more significant $(P<0.05)$ than group $\mathrm{C}$.

\section{Pressure pain threshold}

The within-group analysis revealed a statistically significant improvement in PPT in group B (mean improvement=1.204 $\left.\mathrm{kg} / \mathrm{cm}^{2} ; P<0.05\right)$, but insignificant improvements in groups $\mathrm{A}$ (mean improvement $\left.=0.528 \mathrm{~kg} / \mathrm{cm}^{2} ; P>0.05\right)$ and $\mathrm{C}$ (mean improvement $\left.=0.693 \mathrm{~kg} / \mathrm{cm}^{2} ; P>0.05\right)$ when the follow-up scores at day 15 (PPT 15-PPT 0) were compared with their respective baseline scores. Moreover, all groups except group $\mathrm{C}$ reported a significant improvement $(P<0.05)$ at day 5 postintervention (PPT 5-PPT 0) and at follow-up day 10 (PPT 10-PPT 0). More interestingly, all of the groups had insignificant improvements $(P>0.05)$ immediately postintervention on day 1 (PPT 1-PPT 0).

Between-group comparisons showed insignificant differences $(P>0.05)$ for all time intervals except day 5 postintervention, where group B was significantly different from group $\mathrm{C}(P<0.05)$. Similarly, at follow-up day 15 , a significant difference was reported between groups $\mathrm{A}$ and $\mathrm{B}(P<0.05)$. 
Table 2 Within-group analysis of variables VAS, PPT, ROR, and NDI scores with their mean \pm SD

\begin{tabular}{|c|c|c|c|c|c|c|c|}
\hline \multirow[t]{2}{*}{ Variables } & \multicolumn{5}{|c|}{ VAS (mean $\pm S D)$} & \multirow[t]{2}{*}{ F-value } & \multirow[t]{2}{*}{$P$-value } \\
\hline & VAS 0 & VAS I & VAS 5 & VAS IO & VAS I5 & & \\
\hline Group A & $4.73 \pm 1.79$ & $4.00 \pm 1.36$ & $2.07 \pm 0.59$ & $1.4 \pm 0.63$ & $1.13 \pm 0.74$ & 44.836 & 0.000 \\
\hline Group B & $4.93 \pm 1.39$ & $3.53 \pm 1.46$ & $1.93 \pm 1.39$ & $1.33 \pm 1.23$ & $1.07 \pm 1.16$ & 67.064 & 0.000 \\
\hline \multirow[t]{3}{*}{ Group C } & $4.80 \pm 1.47$ & $3.6 \pm 1.59$ & $2.2 \pm 1.15$ & $2.2 \pm 1.01$ & $1.4 \pm 0.97$ & 32.106 & 0.000 \\
\hline & \multicolumn{5}{|c|}{ PPT (mean $\pm S D)$} & & \\
\hline & PPT 0 & PPT I & PPT 5 & PPT I0 & PPT I5 & & \\
\hline Group A & $0.26 \pm 0.38$ & $0.3 I \pm 0.40$ & $0.73 \pm 0.55$ & $0.91 \pm 0.73$ & $0.77 \pm 0.74$ & $5.74 I$ & 0.000 \\
\hline Group B & $0.15 \pm 0.16$ & $0.44 \pm 0.48$ & $0.92 \pm 0.58$ & $1.03 \pm 0.70$ & $1.35 \pm 0.69$ & 17.591 & 0.000 \\
\hline \multirow[t]{3}{*}{ Group C } & $0.15 \pm 0.28$ & $0.30 \pm 0.43$ & $0.41 \pm 0.39$ & $0.52 \pm 0.48$ & $0.79 \pm 0.68$ & 5.405 & 0.000 \\
\hline & \multicolumn{5}{|c|}{ ROR (mean $\pm S D)$} & & \\
\hline & ROR 0 & ROR I & ROR 5 & ROR I0 & ROR I5 & & \\
\hline Group A & $18.67 \pm 2.80$ & $17.01 \pm 2.89$ & $15.53 \pm 2.29$ & $16.14 \pm 2.72$ & $15.86 \pm 2.72$ & 11.596 & 0.000 \\
\hline Group B & $19.59 \pm 3.00$ & $18.45 \pm 2.29$ & $17.21 \pm 3.05$ & $16.88 \pm 2.65$ & $16.4 \pm 2.83$ & 12.179 & 0.000 \\
\hline \multirow[t]{3}{*}{ Group C } & $17.21 \pm 3.47$ & $16.88 \pm 2.23$ & $15.91 \pm 2.35$ & $|5.5| \pm 2.9 \mid$ & $14.99 \pm 2.21$ & 7.402 & 0.000 \\
\hline & \multicolumn{5}{|c|}{ NDI (mean \pm SD) } & & \\
\hline & NDI 0 & \multicolumn{2}{|l|}{ NDI 5} & \multicolumn{2}{|l|}{ NDI I 5} & & \\
\hline Group A & $21.56 \pm 7.76$ & \multicolumn{2}{|l|}{$11.27 \pm 4.50$} & \multicolumn{2}{|l|}{$6.73 \pm 4.23$} & 29.732 & 0.000 \\
\hline Group B & $22.19 \pm 7.83$ & \multicolumn{2}{|l|}{$I I .4 I \pm 5.56$} & \multicolumn{2}{|l|}{$7.78 \pm 4.83$} & 51.152 & 0.000 \\
\hline Group C & $17.51 \pm 9.57$ & \multicolumn{2}{|l|}{$11.17 \pm 10.82$} & \multicolumn{2}{|l|}{$8.43 \pm 8.23$} & 24.287 & 0.000 \\
\hline
\end{tabular}

Note: The value is significant if $P<0.05$ and highly significant if $P<0.01$.

Abbreviations: NDI, Neck Disability Index; PPT, pressure pain threshold; ROR, range of rotation; SD, standard deviation; VAS, visual analog scale.

Table 3 Between-group analysis of variables VAS, PPT, ROR, and $\mathrm{NDI}$ scores with their $P$-values

\begin{tabular}{|c|c|c|c|c|c|}
\hline Groups & VAS 0 & VAS I & VAS 5 & VAS IO & VAS I 5 \\
\hline$A$ vs $B$ & 0.940 & 0.680 & 0.946 & 0.983 & 0.983 \\
\hline$A$ vs $C$ & 0.993 & 0.760 & 0.946 & 0.100 & 0.759 \\
\hline \multirow[t]{2}{*}{$B$ vs $C$} & 0.973 & 0.992 & 0.801 & 0.068 & 0.650 \\
\hline & PPT 0 & PPT I & PPT 5 & PPT 10 & PPT I5 \\
\hline$A$ vs $B$ & 0.592 & 0.703 & 0.610 & 0.877 & 0.099 \\
\hline$A$ vs $C$ & 0.592 & 1.000 & 0.237 & 0.269 & 1.000 \\
\hline \multirow[t]{2}{*}{ B vs $C$} & 1.000 & 0.691 & $0.033^{*}$ & 0.110 & 0.101 \\
\hline & ROR 0 & ROR I & ROR 5 & ROR I0 & \\
\hline$A$ vs $B$ & 0.722 & 0.433 & 0.218 & 0.765 & 0.851 \\
\hline$A$ vs $C$ & 0.447 & 0.993 & 0.922 & 0.822 & 0.662 \\
\hline \multirow[t]{2}{*}{ B vs $C$} & 0.125 & 0.369 & 0.396 & 0.404 & 0.344 \\
\hline & NDI 0 & \multicolumn{2}{|l|}{ NDI 5} & \multicolumn{2}{|l|}{ NDI I5 } \\
\hline$A$ vs $B$ & 0.979 & \multicolumn{2}{|l|}{0.999} & \multicolumn{2}{|l|}{0.891} \\
\hline$A$ vs $C$ & 0.429 & \multicolumn{2}{|l|}{0.999} & \multicolumn{2}{|l|}{0.743} \\
\hline$B$ vs $C$ & 0.324 & \multicolumn{2}{|l|}{0.996} & \multicolumn{2}{|l|}{0.958} \\
\hline
\end{tabular}

Note: $*$ The value is significant if $P<0.05$ and nonsignificant if $P>0.05$.

Abbreviations: NDI, Neck Disability Index; PPT, pressure pain threshold; ROR, range of rotation; VAS, visual analog scale.

\section{Cervical range of motion (ROR)}

The within-group analysis revealed a statistically significant improvement in ROR in group A (mean improvement $=-2.807 \mathrm{~cm} ; P<0.05)$, group $\mathrm{B}$ (mean improvement $=-3.187$ $\mathrm{cm} ; P<0.05$ ), and group $\mathrm{C}$ (mean improvement $=-2.220 \mathrm{~cm}$; $P<0.05)$ when the follow-up scores at day 15 were compared with their respective baselines scores (ROR 15-ROR 0).
In addition, all groups reported a significant improvement $(P<0.05)$ at follow-up day 10 (ROR 10-ROR 0). Groups A and B showed a significant improvement $(P<0.05)$ at day 5 postintervention (ROR 5-ROR 0), whereas group C did not improve significantly $(P>0.05)$ at day 5 . For the ROR, the experimental group A reported a significant improvement $(P<0.05)$, whereas experimental group B and control group $\mathrm{C}$ reported insignificant improvements $(P>0.05)$ immediately postintervention on day 1 (ROR 1-ROR 0 ).

The between-group comparisons showed statistically insignificant intergroup differences $(P>0.05)$ at all time intervals.

\section{Functional status of the neck (NDI)}

The within-group analysis revealed statistically significant improvement in group A (mean improvement $=-14.829 \%$; $P<0.05$ ), group $\mathrm{B}$ (mean improvement $=-14.409 \% ; P<0.05$ ), and group $\mathrm{C}$ (mean improvement $=-9.081 \% ; P<0.05)$ when the follow-up scores at day 15 (ROR 15-ROR 0) were compared with their respective baselines scores. Statistically significant improvements in postintervention scores at day 5 (ROR 5-ROR 0) were also observed for all groups.

The between-group comparisons showed statistically insignificant intergroup differences $(P>0.05)$ at all time intervals.

\section{Discussion}

This study aimed at assessing and comparing the effectiveness of either type of manual therapy (MPR and MET) for 


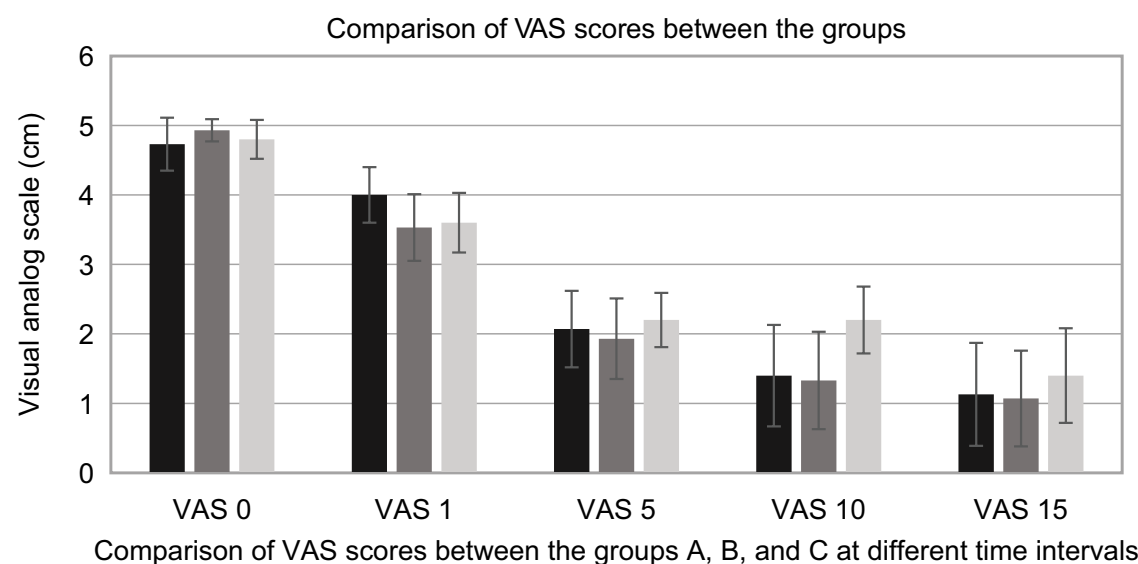

(Day 0, 1, 5, 10 and 15)

- Group A Group B Group C

Figure 2 Comparison of VAS scores (pain intensity) between the groups. Abbreviation: VAS, visual analog scale.

treating neck pain elicited by trigger points. This study examined stress-related (both postural and psychological) neck pain in a younger age group ( $<30$ years), including stressors related to the activities of students and working professionals, such as nursing, physiotherapy, radiology, or desktop and laboratory work. This has been consistently supported by previous studies. ${ }^{26-28}$ A study of nurses observed an association between both physical and psychological exposure in the work environment and seeking care for shoulder or neck pain. ${ }^{27}$ Another study reported that the cumulative effect of postural demands, static holding, and heavy lifting was the work-related cause of neck pain among physiotherapists. ${ }^{29}$ Furthermore, neck problems were found to have an almost $61 \%$ lifetime prevalence among undergraduate physiotherapy students. $^{28}$

The trapezius muscle of women with neck and shoulder myalgia is characterized by its great potential for adaptation following physical exercise. ${ }^{30}$ The significant changes in the number of capillaries and specific changes induced by training at the level of the muscle fibers might well explain the improvements in muscle function. Thus, early physiotherapy interventions with postural awareness, psychological stress reduction, and spinal reconditioning exercises are important for preventing further episodes of neck pain at an early age.

The mean improvement was significant in all three groups in our study. This highlights the effectiveness of our physiotherapy intervention for the management of occupational neck pain, which is mostly neglected among young individuals suffering from such pain. This also highlights the neurophysiological and psychological impact of active neck and shoulder exercises over a short term period of just
15 days, which could help recondition the neck muscles, add to the participants' kinesthetic awareness, and assure the patients that they are being treated. Our results are consistent with those of previous studies that used various types of exercises, such as proprioception, endurance, coordination, strength training and stretching; studies of both low- and high-intensity exercises; and studies that provided supervised departmental or home exercise programs to alleviate neck pain. ${ }^{8,31-34}$ In contrast, a few studies reported an insignificant improvement in neck pain with exercise therapy as an intervention program compared with conservative or no treatment for patients with nonspecific neck pain. ${ }^{11,35}$ Our results are in line with those of a previous study suggesting that improving muscle mechanics and muscle reconditioning can improve work dynamics, with the help of active physiotherapy. ${ }^{36}$

The mean PPT showed improvements in all three groups, but the improvement found in group B was appreciable. This is similar to a study by Leon Chaitow, who demonstrated a decrease in MTrP sensitivity in response to the MET. ${ }^{37}$ The finding that increasing the length of shortened muscles relieved tenderness and pain supports the muscular origin of pain. Stretching exercises have formed the basis of exercise treatment for myofascial pain. ${ }^{37}$ Decreases in pain sensitivity with the MET could be attributed to the inhibition of Ia and IIa afferents from muscle spindles and $\mathrm{Ib}$ afferents from the Golgi tendon organ to the central nervous system. ${ }^{38,39}$ Such an altered afferent drive is thought to influence the activity of alpha motor neurons. This may increase the muscle extensibility and thus decrease tightness, contributing to less pain with pressure and the gradual restoration of normal activity. It could be proposed that stretch equalizes the length 
of the sarcomeres throughout each involved muscle fiber, thus normalizing the function of the contractile elements of the muscle. ${ }^{39}$

Group A showed improvement in the mean PPT score until day 10, after which the improvement disappeared. The increase in PPT scores immediately after the application of MPR has been attributed to the fact that the application of pressure creates a negative pressure, resulting in an increase in vascular flow to the affected area upon release of the pressure. This increase in blood supply cures the energy crisis and oxygen deficit in the shortened sarcomeres following a reduction in the afferent firing of the muscle spindle. ${ }^{40}$ Furthermore, the application of vertical pressure causes elongation of the shortened sarcomeres, gradually returning them to a normal length. This position avoids the stretch reflex and may have decreased muscle sensitivity for a short while by interrupting the afferent discharges of the gamma motor neurons of the muscle spindle; however, long-lasting effects still need to be studied, as the few previous studies examined the immediate effect of this technique. ${ }^{40,41}$ Thus, it remains to be determined whether MPR provides any permanent decrease in tenderness.

There was a marginal improvement in PPT scores in the control group, which received only postural advice and isometric neck exercises. This result concurs with previous studies finding that active home exercise in patients with nonspecific neck pain resulted in a very minor change in PPT scores over a duration of 3-12 months. ${ }^{42}$ However, another group of authors declared that active physiotherapy (exercises for 60 minutes each visit; mean of 13 visits) or passive treatment (heat, massage, and mild stretching for 20 minutes each visit; mean of 10 visits) provided no significant improvement in PPT. ${ }^{43}$ In contrast, one study reported a significant improvement in PPT scores with any kind of exercise training (strengthening, endurance, and coordination) in women with trapezius myalgia. ${ }^{31}$ As the duration of treatment in these previous studies was longer than our intervention program of 15 days, it could be postulated that a minimum number of days of exercise is necessary to reduce the level of tenderness without any manual therapy. This time is required to reverse the pathophysiological processes related to the development of trigger points.

The cervical ROR increased in all three groups. In group $\mathrm{A}$, the improvement occurred just after the first treatment and increased until day 15; group B showed an improvement in ROR beginning on day 5, whereas group $\mathrm{C}$ showed an improvement for the first time on day 10. The later improvement in the ROR in the group receiving the
MET may be due to posttreatment soreness in the first few sessions, which could have interrupted in the active cervical ROR of the subjects; however, this technique was gradually accompanied by myofibrillogenesis and improved stretch tolerance in the following sessions. ${ }^{44}$ An improvement in the control group could be reasonably attributed to the improved biomechanics of the upper body quadrant due to better postural awareness and decreases in pain. This is in contrast to the results of a previous study that demonstrated only minor changes in range of motion with a home exercise program. ${ }^{42}$ However, another study showed significant increases in neck mobility with endurance or strength training for neck pain in women with trapezius myalgia. ${ }^{33}$ Nonetheless, this improvement accompanied stretch exercises in both groups. As our sample consisted of young subjects with no presumed anatomical changes in muscle length, the increase in active ROR in the control group could be credited to an interruption in physiological pain.

Neck disability scores improved in all three groups. Previous studies reported that almost any type of physiotherapy intervention brought about a significant decrease in neck disability and an increase in the functional status of the neck. ${ }^{33,42,45,46}$ It is unclear whether this is merely an effect of time. However, after the termination of passive physiotherapy, the disability scores continued to decrease only in experimental groups. This highlights the subtle role of manual therapy in the treatment of mechanical neck pain syndromes. Accessory manual maneuvers could potentially accentuate the effects of active physiotherapy, particularly for the treatment of pain, bringing about rapid improvement in function. These results also emphasize the psychological support given to the patient by a therapeutic touch.

This study has some limitations. The sample size was small and limited to women. In addition, this study did not analyze the effect of the techniques on advanced objective variables such as electromyography or upper-quadrant posture analysis. Finally, a longer follow-up duration is necessary to analyze the long-term effects of these techniques.

\section{Conclusion}

This study concluded that along with posture correction and active exercises, both the MPR and MET are equally effective for decreasing pain intensity and functional disability of the neck as well as increasing the PPT and cervical range of motion in patients with mechanical pain due to upper trapezius trigger points. Future research should utilize a larger sample size that includes both genders, include advanced variables to analyze technique effects, extend the duration 
of the study and follow-up, and address the duration of pain relief associated with the control group and its contributing factors.

\section{Relevance to clinical practice}

Muscular pain constitutes the largest category of unrecognized and untreated chronic medical problems in clinical practice and is among the most overlooked causes of chronic pain and disability. This study found benefits from the MPT, $\mathrm{MET}$, and postural correction exercises for improving overall pain and function in occupational nonspecific neck pain. The results of this study could aid in determining the most effective treatment for mechanical neck pain due to trigger points.

\section{Acknowledgments}

The authors are grateful to the Deanship of Scientific Research, King Saud University, for funding through the Vice Deanship of Scientific Research Chairs. They also thank the Deanship of Scientific Research and Researchers Support Services Unit at King Saud University for their technical support.

\section{Author contributions}

All the authors contributed to the preparation of the manuscript according to the International Committee of Medical Journal Editors criteria for authorship. They also contributed to the conception of the research design, data collection, data analysis, manuscript formatting, and drafting and critically revising the paper. All of them gave final approval of the version to be published and agreed to be held responsible for all facets of the work.

\section{Disclosure}

The authors report no conflicts of interest in this work.

\section{References}

1. von Korff M, Dworkin SF, Le Resche L. Graded chronic pain status: an epidemiologic evaluation. Pain. 1990;40(3):279-291.

2. Borghouts JA, Koes BW, Vondeling H, Bouter LM. Cost-of-illness of neck pain in the Netherlands in 1996. Pain. 1999;80(3):629-636.

3. Pinto B, Ulman S, Assi H. Prevalence of occupational diseases in information technology industries in Goa. Indian $J$ Occup Environ Med. 2004;8(1):30-33.

4. Ylinen J, Salo P, Nykänen M, Kautiainen H, Häkkinen A. Decreased isometric neck strength in women with chronic neck pain and the repeatability of neck strength measurements. Arch Phys Med Rehabil. 2004;85(8):1303-1308.

5. Falla D, Bilenkij G, Jull G. Patients with chronic neck pain demonstrate altered patterns of muscle activation during performance of a functional upper limb task. Spine. 2004;29(13):1436-1440.

6. Mäkelä M, Heliövaara M, Sievers K, Impivaara O, Knekt P, Aromaa A. Prevalence, determinants, and consequences of chronic neck pain in Finland. Am J Epidemiol. 1991;134(11):1356-1367.
7. Korhonen T, Ketola R, Toivonen R, Luukkonen R, Häkkänen M, Viikari-Juntura E. Work related and individual predictors for incident neck pain among office employees working with video display units. Occup Environ Med. 2003;60(7):475-482.

8. Philadelphia Panel, Experts CS. Philadelphia Panel evidence-based clinical practice guidelines on selected rehabilitation interventions for low back pain. Phys Ther. 2001;81(10):1641-1674.

9. Murphy DR. Cervical Spine Disorders: Conservative Management of Cervical Spine Syndromes. New York, USA: McGraw-Hill Professional; 2000:71-103, 483-513.

10. Van Tulder MW, Goossens ME, Hoving Jl. Non-surgical treatment of chronic neck pain. In: Nachemson AL, Jonsson E, editors. Neck and Back Pain: The Scientific Evidence of Causes, Diagnosis and Treatment. Philadelphia: Lippincott, Williams and Wilkins; 2000:339-354.

11. Swenson RS. Therapeutic modalities in the management of nonspecific neck pain. Phys Med Rehabil Clin N Am. 2003;14(3):605-627.

12. Foster NE, Pincus T, Underwood MR, Vogel S, Breen A, Harding G. Understanding the process of care for musculoskeletal conditions why a biomedical approach is inadequate. Rheumatology. 2003;42(3): 401-404.

13. Korthals-de Bos IB, Müllner M, Hoving JL. Cost effectiveness of physiotherapy, manual therapy, and general practitioner care for neck pain: economic evaluation alongside a randomised controlled trial. Commentary: bootstrapping simplifies appreciation of statistical inferences. BMJ. 2003;326(7395):911-914.

14. Travell JG, Simons DG, Simons LS. Myofascial Pain and Dysfunction: The Trigger Point Manual: Vol. 2: The Lower Extremities. Philadelphia, PA, USA: Lippincott Williams \& Wilkin; 1999:179-187, 237-472.

15. Jensen C, Jansen T, Westgaard RH. EMG of neck and shoulder muscles: the relationship between muscle activity and muscle pain in occupational settings. Kumar S, editor. In: Electromyography in Ergonomics. London: Routledge Taylor \& Francis Group; 2017:227-258.

16. Wolfe F, Clauw DJ, Fitzcharles MA, et al. The American College of Rheumatology preliminary diagnostic criteria for fibromyalgia and measurement of symptom severity. Arthritis Care Res. 2010;62(5):600-610.

17. Simons DG. Diagnostic criteria of myofascial pain caused by trigger points. J Musculoskelet Pain. 1999;7(1-2):111-120.

18. Jensen MP, Karoly P, Braver S. The measurement of clinical pain intensity: a comparison of six methods. Pain. 1986;27(1):117-126.

19. Hawker GA, Mian S, Kendzerska T, French M. Measures of adult pain: Visual Analog Scale for Pain (VAS Pain), Numeric Rating Scale for Pain (NRS Pain), McGill Pain Questionnaire (MPQ), Short-Form McGill Pain Questionnaire (SF-MPQ), Chronic Pain Grade Scale (CPGS), Short Form-36 Bodily Pain Scale (SF-36 BPS), and Measure of Intermittent and Constant Osteoarthritis Pain (ICOAP). Arthritis Care Res. 2011;63(S11):S240-S252.

20. Linde LD, Kumbhare DA, Joshi M, Srbely JZ. The relationship between rate of Algometer application and pain pressure threshold in the assessment of myofascial trigger point sensitivity. Pain Pract. 2018;18(2):224-229.

21. Fischer AA. Algometry in diagnosis of musculoskeletal pain and evaluation of treatment outcome: an update. J Musculoskelet Pain. 1998;6(1):5-32.

22. Antonaci F, Sand T, Lucas GA. Pressure algometry in healthy subjects: inter-examiner variability. Scand J Rehabil Med. 1998;30(1):3-8.

23. Vernon H, Mior S. The Neck Disability Index: a study of reliability and validity. J Manipulative Physiol Ther. 1991;14(7):409-415.

24. Hains F, Waalen J, Mior S. Psychometric properties of the neck disability index. J Manipulative Physiol Ther. 1998;21(2):75-80.

25. Gerwin RD. The taut band and other mysteries of the trigger point: an examination of the mechanisms relevant to the development and maintenance of the trigger point. J Musculoskelet Pain. 2008;16(1-2): 115-121.

26. Engels JA, van der Gulden JW, Senden TF, Hertog CA, Kolk JJ, Binkhorst RA. Physical work load and its assessment among the nursing staff in nursing homes. J Occup Med. 1994;36(3):338-345. 
27. Fredriksson K, Alfredsson L, Ahlberg G, et al. Work environment and neck and shoulder pain: the influence of exposure time. Results from a population based case-control study. Occup Environ Med. 2002;59(3):182-188.

28. Bialocerkowski AE, Grimmer KA, Nyland LJ. Prevalence of and risk factors associated with neck problems in undergraduate physiotherapy students. Phys Canada. 2005;57(4):293-304.

29. Cromie JE, Robertson VJ, Best MO. Work-related musculoskeletal disorders and the culture of physical therapy. Phys Ther. 2002;82(5):459-472.

30. Kadi F, Ahlgren C, Waling K, Sundelin G, Thornell LE. The effects of different training programs on the trapezius muscle of women with work-related neck and shoulder myalgia. Acta Neuropathol. 2000;100(3):253-258.

31. Waling K, Sundelin G, Ahlgren C, Järvholm B. Perceived pain before and after three exercise programs: a controlled clinical trial of women with work-related trapezius myalgia. Pain. 2000;85(1-2):201-207.

32. Jenis LG, Jacquemin J. Conservative management of neck pain. Topics Pain Manage. 2009;25(1):1-7.

33. Ylinen J, Takala EP, Nykänen M, et al. Active neck muscle training in the treatment of chronic neck pain in women: a randomized controlled trial. JAMA. 2003;289(19):2509-2516.

34. Fiebert IM, Roach KE, Cho P, Feigenbaum L, Fong T, Hamer A. The effects of antigravity unsupervised home cervical muscle strengthening protocol on cervical strength in healthy young adults. J Back Musculoskelet Rehabil. 2004;17(2):41-49.

35. Smidt N, de Vet HC, Bouter LM, et al. Effectiveness of exercise therapy: a best-evidence summary of systematic reviews. Aust J Physiother. 2005;51(2):71-85

36. Pearson ND, Walmsley RP. Trial into the effects of repeated neck retractions in normal subjects. Spine. 1995;20(11):1245-1250.
37. Chaitow L. Positional Release Techniques. 4th edition, Churchill Livingstone, Elsevier. 2015.

38. Guissard N, Duchateau J, Hainaut K. Muscle stretching and motoneuron excitability. Eur J Appl Physiol Occup Physiol. 1988;58(1-2):47-52.

39. de Deyne PG. Application of passive stretch and its implications for muscle fibers. Phys Ther. 2001;81(2):819-827.

40. Fryer G, Hodgson L. The effect of manual pressure release on myofascial trigger points in the upper trapezius muscle. J Bodyw Mov Ther. 2005;9(4):248-255.

41. Fernández-de-Las-Peñas C, Alonso-Blanco C, Fernández-Carnero J, Carlos Miangolarra-Page J. The immediate effect of ischemic compression technique and transverse friction massage on tenderness of active and latent myofascial trigger points: a pilot study. J Bodyw Mov Ther. 2006;10(1):3-9.

42. Taimela S, Takala EP, Asklöf T, Seppälä K, Parviainen S. Active treatment of chronic neck pain: a prospective randomized intervention. Spine. 2000;25(8):1021-1027.

43. Levoska S, Keinänen-Kiukaanniemi S. Active or passive physiotherapy for occupational cervicobrachial disorders? A comparison of two treatment methods with a 1-year follow-up. Arch Phys Med Rehabil. 1993;74(4):425-430.

44. Halbertsma JP, Mulder I, Göeken LN, Eisma WH. Repeated passive stretching: acute effect on the passive muscle moment and extensibility of short hamstrings. Arch Phys Med Rehabil. 1999;80(4):407-414.

45. Gross A, Kay TM, Paquin JP. Exercises for mechanical neck disorders. Cochrane Database Syst Rev. 2015;1.

46. Evans R, Bronfort G, Nelson B, Goldsmith CH. Two-year follow-up of a randomized clinical trial of spinal manipulation and two types of exercise for patients with chronic neck pain. Spine. 2002;27(21): 2383-2389.
Journal of Pain Research

\section{Publish your work in this journal}

The Journal of Pain Research is an international, peer reviewed, open access, online journal that welcomes laboratory and clinical findings in the fields of pain research and the prevention and management of pain. Original research, reviews, symposium reports, hypothesis formation and commentaries are all considered for publication.

\section{Dovepress}

The manuscript management system is completely online and includes a very quick and fair peer-review system, which is all easy to use. Visit http://www.dovepress.com/testimonials.php to read real quotes from published authors. 